\title{
PHI density prospectively improves prostate cancer detection
}

\author{
Carsten Stephan $^{1,2} \cdot$ Klaus Jung $^{1,2} \cdot$ Michael Lein $^{3} \cdot$ Hannah Rochow $^{1,2} \cdot$ Frank Friedersdorff $^{1} \cdot$ Andreas Maxeiner $^{1}$
}

Received: 11 August 2020 / Accepted: 29 December 2020 / Published online: 20 January 2021

(c) The Author(s) 2021

\begin{abstract}
Purpose To evaluate the Prostate Health Index (PHI) density (PHID) in direct comparison with PHI in a prospective large cohort.

Methods PHID values were calculated from prostate-specific antigen (PSA), free PSA and [- 2]proPSA and prostate volume. The 1057 patients included 552 men with prostate cancer (PCa) and 505 with no evidence of malignancy (NEM). In detail, 562 patients were biopsied at the Charité Hospital Berlin and 495 patients at the Sana Hospital Offenbach. All patients received systematic or magnetic resonance imaging (MRI)/ultrasound fusion-guided biopsies. The diagnostic accuracy was evaluated by receiver operating characteristic (ROC) curves comparing areas under the ROC-curves (AUC). The decision curve analysis (DCA) was performed with the MATLAB Neural Network Toolbox.

Results PHID provided a significant larger AUC than PHI $(0.835$ vs. $0.801 ; p=0.0013)$ in our prospective cohort of 1057 men from 2 centers. The DCA had a maximum net benefit of 5\% for PHID vs. PHI between 35 and $65 \%$ threshold probability. In those 698 men within the WHO-calibrated PSA grey-zone up to $8 \mathrm{ng} / \mathrm{ml}$, PHID was also significantly better than PHI (AUC 0.819 vs. 0.789; $p=0.0219$ ). But PHID was not different from PHI in the detection of significant PCa.

Conclusions Based on ROC analysis and DCA, PHID had an advantage in comparison with PHI alone to detect any PCa but PHI and PHID performed equal in detecting significant PCa.
\end{abstract}

Keywords Prostate health index (PHI) $\cdot$ PHI density (PHID) $\cdot$ Prostate cancer $\cdot$ Decision curve analysis (DCA)

\section{Introduction}

Prostate-specific antigen (PSA) has a low specificity in the detection of prostate cancer (PCa). In 2010, the Prostate Health Index PHI $([-2]$ proPSA/freePSA $\times \sqrt{ }$ PSA $)$ was implemented [1]. In 2012, the FDA approved PHI for PCa detection and PHI has been established as PCa biomarker [2]. Different multicenter studies [3-5] showed improvement in comparison with percent free PSA (\%fPSA). PHI also identifies clinical significant PCa [6]. PHI further correlates with tumor volume [7] and can predict pathological outcome [8] and tumor recurrence [9] in radical prostatectomy patients. In 2014, the term PHI density (PHID: PHI/prostate

Carsten Stephan

carsten.stephan@charite.de

1 Department of Urology, Charité-Universitätsmedizin Berlin, corporate member of Freie Universität Berlin, Charitéplatz 1, 10117 Berlin, Germany

2 Berlin Institute for Urologic Research, Berlin, Germany

3 Department of Urology, Sana Hospital, Offenbach, Germany volume) was proposed in a study on 275 men including 189 men with no evidence of malignancy (NEM) and $86 \mathrm{PCa}$ patients [10]. The difference in the area under the receiver operating characteristic (ROC)-curve (AUC) between PHI (0.76) and PHID (0.77) was small and multivariate models only marginally improved AUCs to 0.79 [10]. Since then, only few PHID studies were published [11, 12]. Tosoian et al. [12] calculated the largest AUC for PHID with 0.84 while PHI and \%fPSA had 0.76 and 0.75 , respectively. Contrarily, Friedl et al. [11] found a higher AUC for PHI (0.79) than PHID (0.77). Both studies include a relatively low patient number with 112 [11] and 118 men [12].

Aims of this study were:

(i) to investigate the value of PHID to detect any PCa in comparison with $\mathrm{PHI}$ in a large prospective cohort with $>1000$ men and

(ii) to test the diagnostic capacity of PHID in different subgroups and for the detection of clinically significant PCa with Gleason score $\geq 7$. 


\section{Materials and methods}

Based on former patient data from 2002 to 2014 with PSA, free PSA and $[-2]$ proPSA and prostate volume from three surveys $[5,13,14]$, we initiated in 2014 a prospective collection.

Our prospective group consisted of 1057 men with 552 PCa patients (52.2\%) and 505 NEM patients. Between 2014 and 2019, patients were consecutive biopsied within two tertiary hospitals: 562 patients at the Charite Hospital Berlin and 495 patients at the Sana Hospital Offenbach. Magnetic resonance imaging (MRI)/ultrasound fusion-guided biopsies were performed only in Berlin $(n=52$ of $562,9.3 \%)$. Prostate volume was determined by transrectal ultrasound. Detailed exclusion criteria such as prostatitis or others have been applied as described before [5, 14]. The respective hospital ethics committee approved the study. All patients provided written informed consent. Histological results were related to the 2014 proposed ISUP Gleason grading system. A Gleason score $\geq 7 \mathrm{PCa}$ was defined as clinically significant PCa.

Serum samples were prospectively collected and always frozen at $-80{ }^{\circ} \mathrm{C}$ until analysis. The PSA ranged from 0.49 to $25.8 \mathrm{ng} / \mathrm{ml}$ and the PSA calibration was performed based on the WHO PSA reference material. The fully automated immunoassay device Access ${ }^{\circledR}$ (Beckman Coulter, Brea, California) was used for all samples. Measurements of PSA, free PSA and [-2]proPSA were performed in Berlin.

\section{Statistical analysis}

The MedCalc version 15.8 (MedCalc Software, Ostend, Belgium) was used for statistical analysis and ROC analysis. Group differences were assessed by the nonparametric Mann-Whitney $U$ test and correlations were analyzed using the Spearman rank correlation coefficient $\left(r_{\mathrm{s}}\right)$.

Decision curve analysis (DCA) was performed with the MATLAB Neural Network Toolbox (Mathworks) as described earlier [15]. In the DCA, a possible benefit of a marker or model is plotted against threshold probabilities, which then yields the decision curve. The DCA can identify the range of threshold probabilities and the magnitude of benefit, where the marker or model is of value. Two-sided $p$ values $<0.05$ were considered statistically significant.

\section{Results}

The patient characteristic of the cohort is provided in Table 1. All tested parameters differed significantly between both groups, PCa and NEM, respectively. Both, PHI $\left(r_{\mathrm{s}}=0.38\right.$, confidence interval CI 0.31-0.45) and PHID $\left(r_{\mathrm{s}}=0.30\right.$, CI $\left.0.23-0.38\right)$ correlated significantly $(p<0.0001)$ with the Gleason score.

The AUC for PHID (0.835) was significantly larger than the AUC for PHI $(0.801, p=0.0013)$ (Fig. 1a). PHID outperformed all other parameters including PSA (AUC 0.561), PSA density (PSAD, AUC 0.726) and \%fPSA (AUC 0.753, all $p<0.0001$ ). Additionally, the DCA showed an advantage of maximum 4-5\% net benefit for PHID for a broad range between 35 and $65 \%$ threshold probability (Fig. 1b).

The Hybritech-calibrated PSA grey-zone of 2-10 ng/ $\mathrm{ml}$ corresponds to WHO-calibrated values up to $8 \mathrm{ng} / \mathrm{ml}$. A biopsy decision within this specific PSA range is mostly difficult. We therefore additionally analyzed those 698 men with PSA values $1-8 \mathrm{ng} / \mathrm{ml}$. PHID was significantly better than PHI, but the AUC difference was again only 0.03 ( 0.819 vs. $0.789 ; p=0.0219)$ (Table 2 ). In comparison to all patients, the AUCs for PSAD and \%fPSA were only slightly below the AUC of PHI.

A distribution of NEM $(n=505)$ and low risk patients (Gleason score $<7, n=87)$ combined $(n=505+87=593)$ vs. all other PCa (Gleason score $\geq 7, n=465$ ) provided no further improvement of PHID (AUC 0.786) in comparison with PHI (AUC 0.792, $p=0.62$ ). When comparing only the 87 low risk PCa patients (Gleason score $<7$ ) with all other 465 PCa (Gleason score $\geq 7$ ), the AUCs for PHID (0.715) and PHI $(0.74, p=0.27)$ did again not differ from each other.
Table 1 Clinical characteristics (medians and interquartile ranges) of the study cohort

\begin{tabular}{lccrr}
\hline Parameter (medians) & All patients $(n=1057)$ & PCa $(n=552)$ & NEM $(n=505)$ & $p$ value \\
\hline Age (years) & $69(62-73)$ & $67(61-72)$ & $70(64-75)$ & $<0.0001$ \\
Prostate volume $\left(\mathrm{cm}^{3}\right)$ & $47(34-66)$ & $39(29-52)$ & $59(44-80)$ & $<0.0001$ \\
PSA (ng/ml) & $6.41(4.33-9.24)$ & $6.68(4.74-9.36)$ & $6.02(3.74-9.12)$ & 0.0006 \\
\%fPSA (\%) & $14.9(10.5-21.5)$ & $12.0(8.84-16.5)$ & $18.9(14.2-25.3)$ & $<0.0001$ \\
PSAD & $0.135(0.09-0.21)$ & $0.17(0.11-0.26)$ & $0.1(0.06-0.15)$ & $<0.0001$ \\
PHI & $52.3(38.1-72.5)$ & $65.3(50.2-87.7)$ & $40.4(30.6-53.9)$ & $<0.0001$ \\
PHID & $1.1(0.62-1.98)$ & $1.73(1.12-2.61)$ & $0.66(0.44-1.01)$ & $<0.0001$ \\
\hline
\end{tabular}

PSA prostate-specific antigen, \%fPSA percent free PSA, PSAD PSA density, PHI Prostate Health Index, PHID PHI density 
Fig. 1 The prospective group with 1057 patients with a receiver operating characteristic for PSA (AUC: 0.561), PSAD (0.726), PHI (0.801) and PHID (0.835) and with b decision curve analysis (DCA) comparing model 1 using PHI with model 2 using PSAD with model 3 using PHID, to biopsyall and biopsy-none strategies (a)

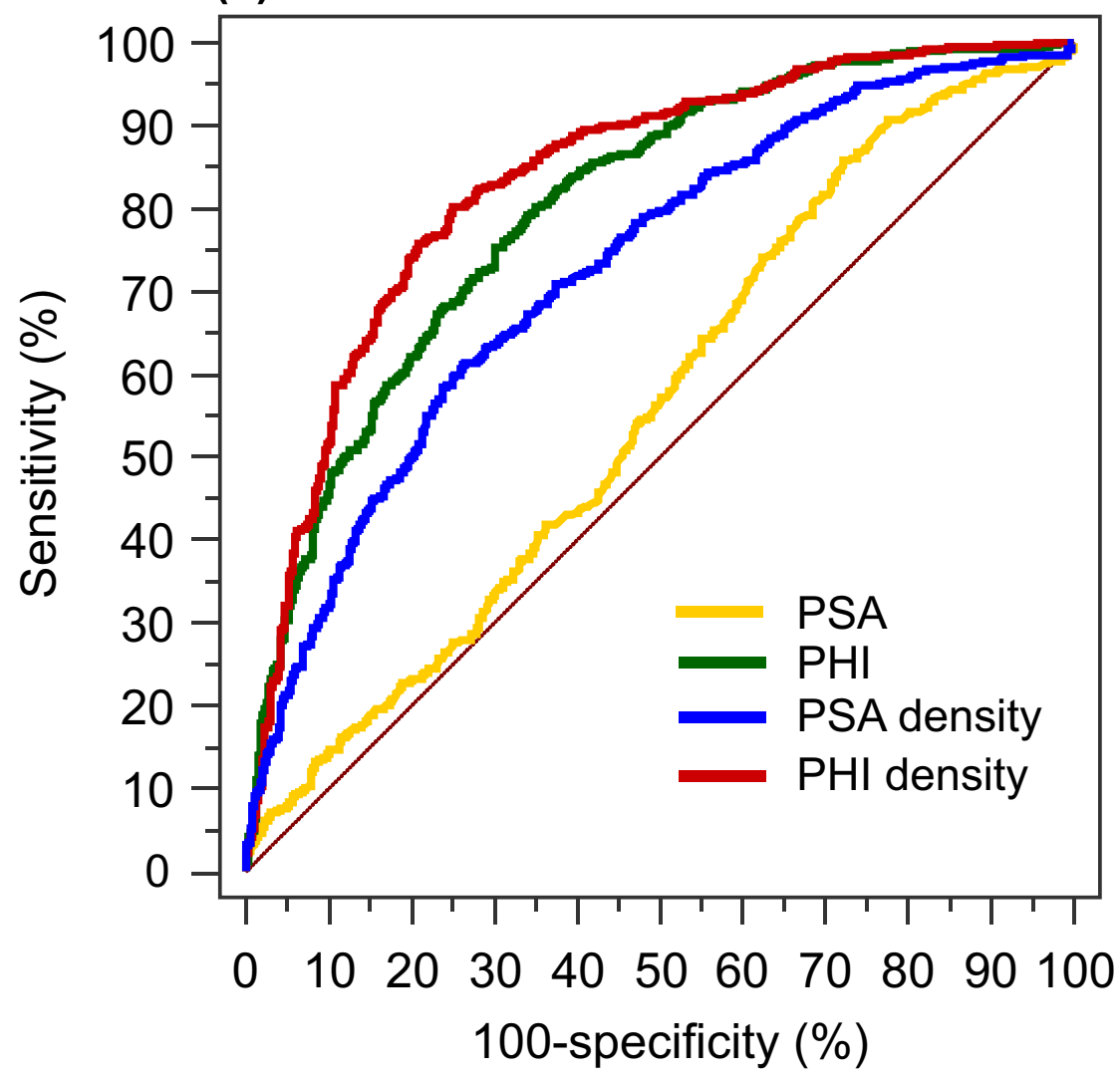

(b)

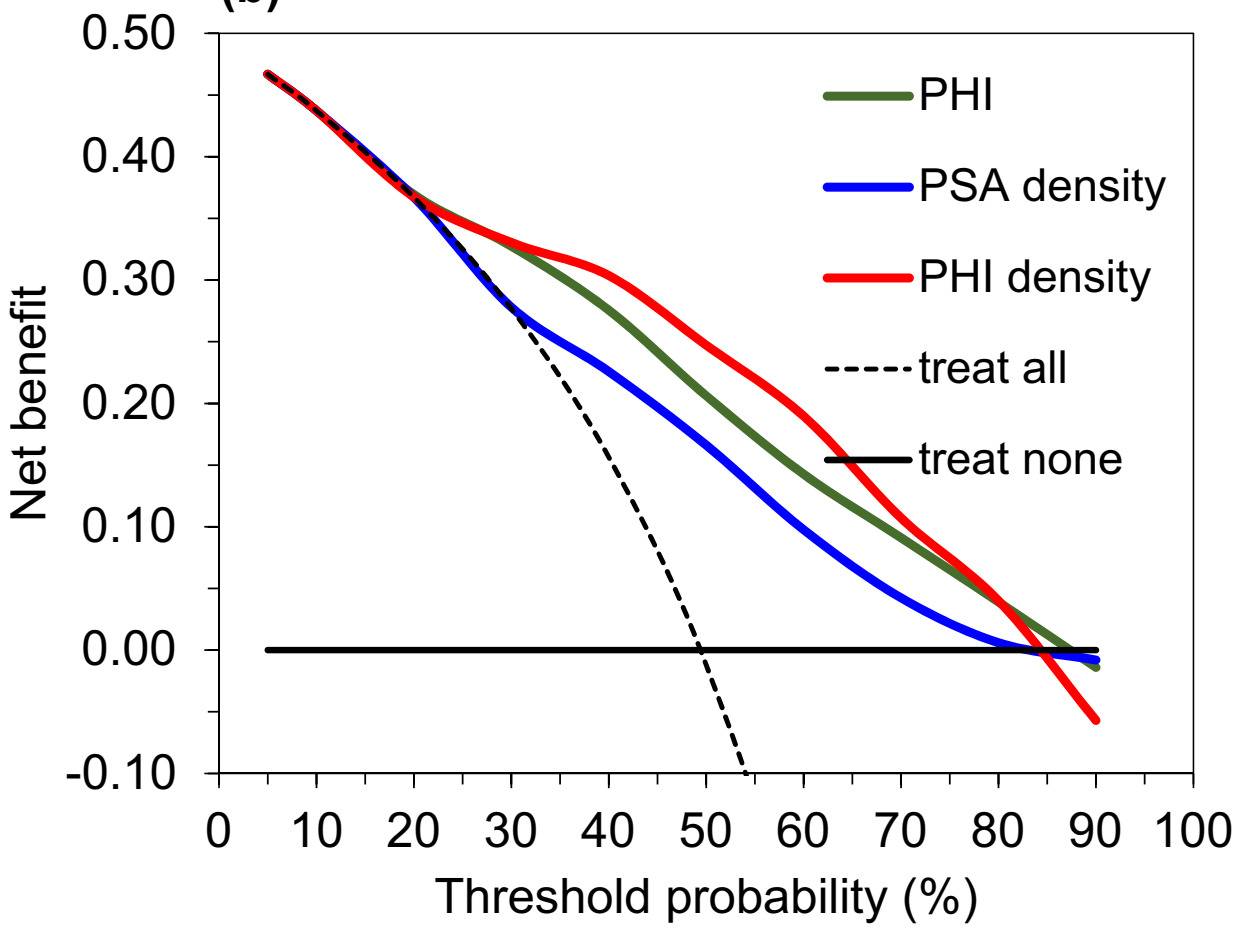


Table 2 AUC comparison of the cohort with PSA values $1-8 \mathrm{ng} / \mathrm{ml}$ in $n=698$ patients

\begin{tabular}{llll}
\hline Parameter & AUC & $\begin{array}{l}\text { 95\% confidence } \\
\text { interval }\end{array}$ & $p$ value versus PHI \\
\hline PSA & 0.591 & $0.55-0.63$ & $<0.0001$ \\
\%fPSA & 0.748 & $0.71-0.78$ & 0.0377 \\
PSAD & 0.749 & $0.72-0.78$ & 0.0361 \\
PHI & 0.789 & $0.76-0.82$ & - \\
PHID & 0.819 & $0.79-0.85$ & 0.0219 \\
\hline
\end{tabular}

$A U C$ area under the ROC curve, PSA prostate-specific antigen, $\%$ FPSA percent free PSA, PSAD PSA density, PHI Prostate Health Index, PHID PHI density

Different prostate volume cutoffs were also evaluated but no improvements for PHID were found. For those 408 patients with small volumes $\leq 40 \mathrm{~cm}^{3}$, PHI and PHID were not significantly different between PCa and NEM (AUCs: 0.729 vs. $0.721, p=0.58)$. Within this group with prostate volume $\leq 40 \mathrm{~cm}^{3}$ the Gleason score $\leq 6 \mathrm{PCa}(n=38)$ as well as the NEM $(n=99)$ together $(n=137)$ provided also no difference in comparison with the Gleason score $\geq 7 \mathrm{PCa}$ $(n=271)$ regarding PHI and PHID (AUCs: 0.74 vs. 0.736, $p=0.77)$. The same was visible when comparing the 38 low risk PCa patients (Gleason score $<7$ ) with the 271 Gleason score $\geq 7 \mathrm{PCa}$ (AUCs: 0.74 vs. $0.736, p=0.77$ ). The remaining 649 patients (volume $>40 \mathrm{~cm}^{3}$ ) also showed no difference between PHID (AUC: 0.82) and PHI $(0.802, p=0.15)$.

\section{Discussion}

Biomarkers for PCa detection play an important role and PHI is able to further improve specificity over PSA and \% fPSA $[16,17]$. While one survey in 112 men found a higher AUC for PHI (0.79) than for PHID (0.77) [11], others showed in 275 and 118 men a further improvement when using PHID (0.77 or 0.84 ) instead of PHI only (each 0.76 ) [10, 12].

The results of our large prospective cohort confirm a further advantage using PHID in comparison with PHI. The absolute AUC difference between 0.8 for PHI and 0.835 for PHID in our group (Fig. 1a) is small but significant. The same AUC advantage for PHID (0.819) vs. PHI (0.789) is visible within the PSA grey-zone $1-8 \mathrm{ng} / \mathrm{ml}$ (Table 2). More importantly, the DCA revealed an identical advantage for PHID between 35 and $65 \%$ threshold probability (Fig. 1b) in the whole cohort and within the PSA grey-zone (data not shown). The importance of a DCA net benefit in relation to an improved AUC has been discussed elsewhere [18].

Our data are in line with the results of Tosoian et al. [12], where the authors claimed in respect to their 118 patients, that PHID has the strongest discriminative ability for clinically significant PCa with an AUC of 0.84. In our significantly larger study group with 1057 patients, PHID had a similar AUC of 0.835 . Thus, PHID currently represents the best discriminative value for $\mathrm{PCa}$ detection. However, PHI alone did not differ from PHID in any AUC comparison with different volume cutoffs nor in detecting significant Gleason score $\geq 7$ PCa. This shows the excellent discriminatory power of $\mathrm{PHI}$ independently from prostate volume, which has been confirmed in a recent study in more than 1600 Asian men [19]. Our data confirmed the earlier described phenomenon by Friedl et al. [11], where the AUC for PHI (0.79) was reported higher than the AUC of PHID (0.77). A subgroup analysis within our study based on various prostate volume cutoffs found always similar AUCs for PHI in comparison with PHID without statistical differences. Because PHID includes prostate volume, subgroup analyses with either selected small or relatively large glands might be responsible for somewhat lower AUCs for PHID in comparison with PHI. PHI might also be the preferred parameter in small glands $\leq 40 \mathrm{~cm}^{3}$ because PPA as part of the PHI formula showed a better discriminatory power in these patients [20]. Additionally, a smaller number of patients $(n=112)$ in the mentioned study [11] might also influence results in favor for PHI despite this phenomenon has been recently also partially described in a large cohort [21].

A comparison of PHI with another current prostate biomarker, the four-kallikrein panel showed comparable results [22]. However, there is a difference for a possible routine use between the FDA approved PHI with its availability in hospitals and reference laboratories and the four-kallikrein panel, which is not approved and only available as a sendout to the company. An assessment of the four-kallikrein panel together with prostate volume in a large group is still lacking.

PHID $\left(r_{\mathrm{s}}=0.30\right)$ further showed a weaker but still significant correlation to Gleason score in comparison with PHI $\left(r_{\mathrm{s}}=0.38\right)$. This is in congruence with initial \%[-2]proPSA density $\left(r_{\mathrm{s}}=0.205, p=0.05\right)$ and PHI $\left(r_{\mathrm{s}}=0.22, p=0.039\right)$ data, where the density value correlated also weaker than PHI with the Gleason score [10].

Conformingly, PHID could not improve the detection of clinically significant PCa with Gleason score $\geq 7$ in comparison with NEM and Gleason score $<7$ combined in our prospective cohort. This phenomenon with no improvement for clinically significant PCa has been earlier described for PHI in smaller cohorts, too [23, 24]. PHI was also not able to detect significant PCa with Gleason score $\geq 7$ [23, 24]. Contrary, in earlier large studies PHI could preferentially detect aggressive $\mathrm{PCa}[3-5,25]$. A recent nomogram using $\mathrm{PHI}$ and prostate volume also detected aggressive $\mathrm{PCa}$ [26].

Druskin et al. [27] combined PHID with MRI and prior negative biopsy status in 241 patients for the diagnosis of clinically significant PCa. Their PHID medians were 1.18 and 0.55 in men with and without clinically significant 
$\mathrm{PCa}$ [27]. These absolute PHID medians are different from our 698 patients (356 PCa and 342 NEM) with PSA values $<8 \mathrm{ng} / \mathrm{ml}$ with 1.63 for any PCa and 0.67 for NEM. Possible reasons for our higher values might be no prior biopsies and no MRI fusion biopsies. The inclusion of MRI data with the Prostate Imaging Reporting and Data System (PI-RADS) score into multivariable models seems to be reasonable and potentially further enables PCa diagnostics with an AUC increase from 0.78 (all 241 men) to 0.90 (subgroup of 104 men with MRI) for PHID [27]. In 2014, Porpiglia et al. [28] first combined MRI, PHI and PCA3 in 170 patients. They reported very high AUC values $>0.9$ but the PI-RADS scoring was not used [28]. Currently, most patients already present a suspicious MRI for biopsy decision. PHI and PHID should be used in cases of a not suspicious MRI and continuously elevated PSA values. Only when MRI and PHI are not suspicious, a biopsy should be avoided.

Recently, a study by Lopes Vendrami et al. [29] on 211 men with PHID results and at least one suspected MRI lesion PI-RADS $\geq 3$ was published. PHI and PHID showed comparable AUCs of 0.78 and 0.82 with our data and the authors further concluded that the use of MRI/ultrasound fusionguided biopsies in comparison to systematic biopsies may have favored the results for PHID [29]. Most recently, Hsieh et al. [30] combined not PHID but PHI with MRI results in 102 men and they found an improved AUC from 0.735 (only PHI) to 0.873 . As a weakness of our study, we had only 52 patients with fusion biopsies. Despite neither PHI (48.5) nor PHID (1.01) were different from those patients without fusion biopsies (46.3 and 0.94), further recommendations based on our data cannot be given. As further limitation, we did not collect information on family history on PCa and we did also not include the digital rectal exam into analysis.

Finally, this prospective study with more than 1000 patients confirms the initial hypothesis from 2014, that prostate dimension-adjusted PSA subforms may better differentiate between PCa and NEM patients and that especially PHID offers a gain in accuracy with respect to PSA, PSAD, \%fPSA and PHI [10]. While Mearini et al. [10] found in their prospective group of 275 men with PSA values of $2-10 \mathrm{ng} / \mathrm{ml}$ including 26 PCa patients with Gleason score $\geq 7$ an AUC gain between 0.05 and 0.08 in six different models to detect significant $\mathrm{PCa}$, we could not confirm this advantage. In our much larger cohort with $84 \%(n=465)$ Gleason score $\geq 7$ PCa patients no significant difference between PHID (AUC 0.786 ) and PHI alone (AUC 0.792, $p=0.62$ ) was visible.

\section{Conclusions}

Our data could confirm a significant advantage for PHID in comparison with $\mathrm{PHI}$ alone in detecting any $\mathrm{PCa}$. But $\mathrm{PHI}$ alone also reaches a high discriminatory power with no difference from PHID in detecting significant PCa with Gleason score $\geq 7$. However, in line with the most recent study [29], we also recommend using both, prostate volume and PHI due to an improved diagnostic efficacy in PCa detection with the combined value PHID.

Acknowledgements We thank Silke Rabenhorst and Sabine Becker for technical assistance. We thank Dr. Klaus und Renate Schreiber for financial support to obtain PSA, fPSA and p2PSA test kits.

Author contributions CS: project development, data management, manuscript writing. KJ: data analysis, manuscript editing. ML: data collection, manuscript editing. HR: data collection, data analysis. FF: data collection, manuscript editing. AM: project development, manuscript editing, manuscript overview.

Funding Open Access funding enabled and organized by Projekt DEAL.

\section{Compliance with ethical standards}

Conflict of interest The authors declare that they have no conflict of interest.

Research involving human participants The study was approved by the institutional research ethics committee.

Informed consent Informed consent was obtained from all individual participants included in the study.

Open Access This article is licensed under a Creative Commons Attribution 4.0 International License, which permits use, sharing, adaptation, distribution and reproduction in any medium or format, as long as you give appropriate credit to the original author(s) and the source, provide a link to the Creative Commons licence, and indicate if changes were made. The images or other third party material in this article are included in the article's Creative Commons licence, unless indicated otherwise in a credit line to the material. If material is not included in the article's Creative Commons licence and your intended use is not permitted by statutory regulation or exceeds the permitted use, you will need to obtain permission directly from the copyright holder. To view a copy of this licence, visit http://creativecommons.org/licenses/by/4.0/.

\section{References}

1. Jansen FH, van Schaik RH, Kurstjens J, Horninger W, Klocker $\mathrm{H}$, Bektic J, Wildhagen MF, Roobol MJ, Bangma CH, Bartsch G (2010) Prostate-specific antigen (PSA) isoform p2PSA in combination with total PSA and free PSA improves diagnostic accuracy in prostate cancer detection. Eur Urol 57:921-927

2. Ferro M, De Cobelli O, Lucarelli G, Porreca A, Busetto GM, Cantiello F, Damiano R, Autorino R, Musi G, Vartolomei MD, Muto M, Terracciano D (2020) Beyond PSA: the role of Prostate Health Index (phi). Int J Mol Sci 21:1184. https://doi.org/10.3390/ ijms21041184

3. Catalona WJ, Partin AW, Sanda MG, Wei JT, Klee GG, Bangma CH, Slawin KM, Marks LS, Loeb S, Broyles DL, Shin SS, Cruz AB, Chan DW, Sokoll LJ, Roberts WL, van Schaik RH, Mizrahi IA (2011) A multicenter study of [-2]pro-prostate specific antigen 
combined with prostate specific antigen and free prostate specific antigen for prostate cancer detection in the 2.0 to $10.0 \mathrm{ng} / \mathrm{ml}$ prostate specific antigen range. J Urol 185:1650-1655

4. Lazzeri M, Haese A, de la Taille A, Palou RJ, McNicholas T, Lughezzani G, Scattoni V, Bini V, Freschi M, Sussman A, Ghaleh B, Le CP, Alberola BJ, Esquena FS, Graefen M, Guazzoni G (2013) Serum isoform [-2]proPSA derivatives significantly improve prediction of prostate cancer at initial biopsy in a total PSA range of 2-10 ng/ml: a multicentric European study. Eur Urol 63:986-994

5. Stephan C, Vincendeau S, Houlgatte A, Cammann H, Jung K, Semjonow A (2013) Multicenter evaluation of [-2]proprostatespecific antigen and the prostate health index for detecting prostate cancer. Clin Chem 59:306-314

6. Loeb S, Sanda MG, Broyles DL, Shin SS, Bangma CH, Wei JT, Partin AW, Klee GG, Slawin KM, Marks LS, van Schaik RH, Chan DW, Sokoll LJ, Cruz AB, Mizrahi IA, Catalona WJ (2015) The prostate health index selectively identifies clinically significant prostate cancer. J Urol 193:1163-1169

7. Friedersdorff F, Gross B, Maxeiner A, Jung K, Miller K, Stephan C, Busch J, Kilic E (2017) Does the prostate health index depend on tumor volume? - a study on 196 patients after radical prostatectomy. Int J Mol Sci 18:E488

8. Guazzoni G, Lazzeri M, Nava L, Lughezzani G, Larcher A, Scattoni V, Gadda GM, Bini V, Cestari A, Buffi NM, Freschi M, Rigatti P, Montorsi F (2012) Preoperative prostate-specific antigen isoform p2PSA and its derivatives, \%p2PSA and prostate health index, predict pathologic outcomes in patients undergoing radical prostatectomy for prostate cancer. Eur Urol 61:455-466

9. Maxeiner A, Kilic E, Matalon J, Friedersdorff F, Miller K, Jung K, Stephan C, Busch J (2017) The prostate health index PHI predicts oncological outcome and biochemical recurrence after radical prostatectomy - analysis in 437 patients. Oncotarget 8:79279-79288

10. Mearini L, Ferri C, Lazzeri M, Bini V, Nunzi E, Fiorini D, Costantini E, Manasse GC, Porena M (2014) Evaluation of prostatespecific antigen isoform p2PSA and its derivates, \%p2PSA, prostate health index and prostate dimension-adjusted related index in the detection of prostate cancer at first biopsy: an exploratory, prospective study. Urol Int 93:135-145

11. Friedl A, Stangl K, Bauer W, Kivaranovic D, Schneeweiss J, Susani M, Hruby S, Lusuardi L, Lomoschitz F, Eisenhuber-Stadler E, Schima W, Brossner C (2017) Prostate-specific antigen parameters and Prostate Health Index enhance prostate cancer prediction with the in-bore 3-t magnetic resonance imaging-guided transrectal targeted prostate biopsy after negative 12-core biopsy. Urology 110:148-153

12. Tosoian JJ, Druskin SC, Andreas D, Mullane P, Chappidi M, Joo S, Ghabili K, Mamawala M, Agostino J, Carter HB, Partin AW, Sokoll LJ, Ross AE (2017) Prostate Health Index density improves detection of clinically significant prostate cancer. BJU Int 120:793-798

13. Stephan C, Kahrs AM, Cammann H, Lein M, Schrader M, Deger S, Miller K, Jung K (2009) A [-2]proPSA-based artificial neural network significantly improves differentiation between prostate cancer and benign prostatic diseases. Prostate 69:198-207

14. Stephan C, Lein M, Matalon J, Kilic E, Zhao Z, Busch J, Jung K (2016) Serum Vitamin D is not helpful in predicting Prostate Cancer aggressiveness compared with the Prostate Health Index. J Urol 196:709-714

15. Stephan C, Jung K, Semjonow A, Schulze-Forster K, Cammann $\mathrm{H}, \mathrm{Hu} \mathrm{X}$, Meyer HA, Bogemann M, Miller K, Friedersdorff F (2013) Comparative assessment of urinary prostate cancer antigen 3 and TMPRSS2:ERG gene fusion with the serum [-2]proprostate-specific antigen-based prostate health index for detection of prostate cancer. Clin Chem 59:280-288
16. Filella X, Foj L (2018) Novel biomarkers for prostate cancer detection and prognosis. Adv Exp Med Biol 1095:15-39

17. Lazzeri M, Lughezzani G, Haese A, McNicholas T, de la Taille A, Buffi NM, Cardone P, Hurle R, Casale P, Bini V, Redorta JP, Graefen M, Guazzoni G (2016) Clinical performance of prostate health index in men with tPSA $>10 \mathrm{ng} / \mathrm{ml}$ : results from a multicentric European study. Urol Oncol 34:415-419

18. Steyerberg EW, Pencina MJ, Lingsma HF, Kattan MW, Vickers AJ, Van CB (2012) Assessing the incremental value of diagnostic and prognostic markers: a review and illustration. Eur J Clin Invest 42:216-228

19. Huang D, Wu YS, Ye DW, Qi J, Liu F, Helfand BT, Zheng SL, Ding Q, Xu DF, Na R, Xu JF, Sun YH (2020) Prostate volume does not provide additional predictive value to prostate health index for prostate cancer or clinically significant prostate cancer: results from a multicenter study in China. Asian J Androl 22:539-543

20. Stephan C, Lein M, Jung K, Schnorr D, Loening SA (1997) The influence of prostate volume on the ratio of free to total prostate specific antigen in serum of patients with prostate carcinoma and benign prostate hyperplasia. Cancer 79:104-109

21. Chiu PK, Ng CF, Semjonow A, Zhu Y, Vincendeau S, Houlgatte A, Lazzeri M, Guazzoni G, Stephan C, Haese A, Bruijne I, Teoh JY, Leung CH, Casale P, Chiang CH, Tan LG, Chiong E, Huang CY, Wu HC, Nieboer D, Ye DW, Bangma CH, Roobol MJ (2019) A multicentre evaluation of the role of the Prostate Health Index (PHI) in regions with differing prevalence of prostate cancer: adjustment of PHI reference ranges is needed for european and asian settings. Eur Urol 75:558-561

22. Nordstrom T, Vickers A, Assel M, Lilja H, Gronberg H, Eklund M (2015) Comparison between the four-kallikrein panel and prostate health index for predicting prostate cancer. Eur Urol 68:139-146

23. Sanchis-Bonet A, Barrionuevo-Gonzalez M, Bajo-Chueca AM, Pulido-Fonseca L, Ortega-Polledo LE, Tamayo-Ruiz JC, SanchezChapado M (2018) Validation of the prostate health index in a predictive model of prostate cancer. Actas Urol Esp 42:25-32

24. Guazzoni G, Nava L, Lazzeri M, Scattoni V, Lughezzani G, Maccagnano C, Dorigatti F, Ceriotti F, Pontillo M, Bini V, Freschi M, Montorsi F, Rigatti P (2011) Prostate-specific antigen (PSA) isoform p2PSA significantly improves the prediction of prostate cancer at initial extended prostate biopsies in patients with total PSA between 2.0 and $10 \mathrm{ng} / \mathrm{ml}$ : results of a prospective study in a clinical setting. Eur Urol 60:214-222

25. Loeb S, Shin SS, Broyles DL, Wei JT, Sanda M, Klee G, Partin AW, Sokoll L, Chan DW, Bangma CH, van Schaik RHN, Slawin KM, Marks LS, Catalona WJ (2017) Prostate Health Index improves multivariable risk prediction of aggressive prostate cancer. BJU Int 120:61-68

26. Foj L, Filella X (2020) Development and internal validation of a novel PHI-nomogram to identify aggressive prostate cancer. Clin Chim Acta 501:174-178

27. Druskin SC, Tosoian JJ, Young A, Collica S, Srivastava A, Ghabili K, Macura KJ, Carter HB, Partin AW, Sokoll LJ, Ross AE, Pavlovich CP (2018) Combining Prostate Health Index density, magnetic resonance imaging and prior negative biopsy status to improve the detection of clinically significant prostate cancer. BJU Int 121:619-626

28. Porpiglia F, Russo F, Manfredi M, Mele F, Fiori C, Bollito E, Papotti M, Molineris I, Passera R, Regge D (2014) The roles of multiparametric magnetic resonance imaging, PCA3 and prostate health index-which is the best predictor of prostate cancer after a negative biopsy? J Urol 192:60-66

29. Lopes Vendrami C, McCarthy RJ, Chatterjee A, Casalino D, Schaeffer EM, Catalona WJ, Miller FH (2019) The utility of prostate specific antigen density, prostate health index and prostate health index density in predicting positive prostate biopsy 
outcome is dependent on the prostate biopsy methods. Urology 129:159

30. Hsieh PF, Li WJ, Lin WC, Chang H, Chang CH, Huang CP, Yang CR, Chen WC, Chang YH, Wu HC (2020) Combining prostate health index and multiparametric magnetic resonance imaging in the diagnosis of clinically significant prostate cancer in an Asian population. World J Urol 38:1207-1214
Publisher's Note Springer Nature remains neutral with regard to jurisdictional claims in published maps and institutional affiliations. 